First principles simulation of a superionic phase of hydrogen fluoride (HF) at high pressures and temperatures

Nir Goldman, Laurence E. Fried

April 13, 2006

Journal of Chemical Physics 
This document was prepared as an account of work sponsored by an agency of the United States Government. Neither the United States Government nor the University of California nor any of their employees, makes any warranty, express or implied, or assumes any legal liability or responsibility for the accuracy, completeness, or usefulness of any information, apparatus, product, or process disclosed, or represents that its use would not infringe privately owned rights. Reference herein to any specific commercial product, process, or service by trade name, trademark, manufacturer, or otherwise, does not necessarily constitute or imply its endorsement, recommendation, or favoring by the United States Government or the University of California. The views and opinions of authors expressed herein do not necessarily state or reflect those of the United States Government or the University of California, and shall not be used for advertising or product endorsement purposes. 


\title{
First principles simulation of a superionic phase of hydrogen fluoride (HF) at high pressures and temperatures
}

\author{
Nir Goldman* and Laurence E. Fried
}

April 10, 2006

Classification: Physical Sciences

PACS: 31.15Ar, 61.20Ja, 71.15Pd, 62.50+p

Word Count:

Number of text pages (including references and figures), figures, and tables

Chemistry and Materials Sciences Directorate, Lawrence Livermore

National Laboratory, P. O. Box 808, Livermore, California 94550

\footnotetext{
${ }^{*}$ Corresponding author. E-mail: goldman14@llnl.gov
} 


\begin{abstract}
We have conducted $A b$ initio molecular dynamics simulations of hydrogen fluoride $(\mathrm{HF})$ at pressures of 5-66 GPa along the $900 \mathrm{~K}$ isotherm. We predict a superionic phase at $33 \mathrm{GPa}$, where the fluorine atoms are fixed in a bcc lattice while the hydrogen atoms diffuse rapidly with a diffusion constant of between $2 \times 10^{-5}$ and $5 \times 10^{-5}$ $\mathrm{cm}^{2} / \mathrm{s}$. We find that a transformation from asymmetric to symmetric hydrogen bonding occurs in HF at $66 \mathrm{GPa}$ and 900K. With superionic HF we have discovered a model system where symmetric hydrogen bonding occurs at experimentally achievable conditions. Given previous results on superionic $\mathrm{H}_{2} \mathrm{O}[1,2,3]$ and $\mathrm{NH}_{3}[1]$, we conclude that high $\mathrm{P}, \mathrm{T}$ superionic phases of electronegative element hydrides could be common.
\end{abstract}

Superionic solids exhibit exceptionally high ionic conductivity, where one ion type rapidly diffuses through a crystalline lattice of the remaining types. This is a unique phase of matter in which bonds are breaking and reforming very rapidly. Since their discovery in 1836, a fundamental understanding of superionic conductors has been one of the major challenges in condensed matter physics[4]. In general, it has been difficult to create a simple set of rules governing superionic phases. Studies have mostly been limited to compounds with metallic elements, such as metal halides like $\mathrm{AgI}$ and $\mathrm{PbF}_{2}[4]$. In contrast, the existence of superionic solid phases of hydrogen bonded compounds has been theorized previously[5, 1]. Recent experimental and computational results indicate the presence of a high pressure triple point in the $\mathrm{H}_{2} \mathrm{O}$ phase diagram $[6,7,2]$, including a so-called superionic solid phase with fast hydrogen diffusion $[2,3]$.

Cavazzoni et al.[1] found a symmetrization of the H-O-H hydrogen bond in superionic water. More recently, Goldman et al. found that the superionic phase of water undergoes a transformation from asymmetric (conventional) to symmetric hydrogen bonding at pressures above $95 \mathrm{GPa}$ at $2000 \mathrm{~K}$. In symmetric hydrogen bonding, the intramolecular $\mathrm{X}-\mathrm{H}$ bond becomes identical to the intermolecular $\mathrm{X}-\mathrm{H}$ bond, where $\mathrm{X}$ is an electronegative element. This provides a depression in the bond mid-point energetic barrier, which facilitates pressure induced bond dissociation[8]. Consequently, symmetric hydrogen bonding could be a key factor in superionic diffusion in hydrogen bonding systems.

Due to current limitations in diamond anvil cell techniques, the temperatures and pressures that can be investigated experimentally are too low to 
probe the predicted transformation to symmetric hydrogen bonding in previously studied hydrides, such as $\mathrm{NH}_{3}[1]$ and $\mathrm{H}_{2} \mathrm{O}$. For instance, in water Goldman et al.[3] predicted that symmetric hydrogen bonding occurs at 95 $\mathrm{GPa}$ and $2000 \mathrm{~K}$. The combination of very high pressure and temperature make it challenging to use traditional optical probes in the diamond anvil cell[2]. Thus, we seek to find other materials that will achieve a symmetrically hydrogen bonded superionic phase at more accessible temperatures and pressures. We expect HF to demonstrate symmetric hydrogen bonding at lower pressures and temperatures than $\mathrm{H}_{2} \mathrm{O}$ due to the high strength of its hydrogen bonds.

Although HF clusters have been studied extensively (e. g., ref. [9]), relatively little is known about the condensed phase. In particular, few high pressure studies of HF exist, despite the relative simplicity of the molecule. A previous Raman study of solid HF obtained spectra at $25 \mathrm{~K}$ and up to $17 \mathrm{GPa}[10]$. The $\mathrm{F}-\mathrm{H}$ stretch showed red-shifting and disappearance (at ca. $7 \mathrm{GPa}$ ) as a function of pressure, concomitant with blue-shifting and broadening of the librational modes. These features were attributed to the symmetrization of the hydrogen bond, as observed in other hydrides at high pressure, including $\mathrm{DCl}[11], \mathrm{HBr}[12]$ and $\mathrm{H}_{2} \mathrm{O}[2]$. This observation suggests the existence of a superionic phase of HF at previously uninvestigated pressures and temperatures.

As a result, HF provides a unique system for studying pressure and temperature induced effects on hydrogen bonded systems. It forms some of the strongest hydrogen bonds known[13, 14], making it an ideal material for our purposes. We report results of Car-Parrinello molecular dynamics simulations[15] along the $900 \mathrm{~K}$ isotherm from 5 - 66 GPa. In these simulations we observe a transformation from a molecular solid to one which exhibits superionic diffusion mediated by symmetric hydrogen bonds at experimentally achievable conditions. Our results show that symmetric hydrogen bonding could play a large role in this superionic diffusion. The lower pressures needed to induce this phase of HF will allow for more extensive experimental studies of superionic phases of hydrogen bonded compounds. Our results indicate that previously undiscovered superionic solids, e. g. $\mathrm{HCl}$ and $\mathrm{HBr}$, may also exist at experimentally accessible temperatures and pressures. 


\section{Methods}

For our simulations we have used Car-Parrinello Molecular Dynamics[15]. We used the BLYP exchange-correlation functional[16, 17], and Troullier-Martins pseudo-potentials[18] for both fluorine and hydrogen. In order to insure convergence of the stress tensor, we used a plane wave cutoff of 180 Rydberg. The temperature was controlled by using Nosé-Hoover thermostats[19, 20] for all nuclear degrees of freedom. A fictitious electron mass of 200 au was used. This resulted in a time step of $0.048 \mathrm{fs}$. The simulations were performed on $64 \mathrm{HF}$ molecules in a fixed volume cell with periodic boundary conditions.

Our simulations began from an initial state of crystalline HF. The crystal structure of $\mathrm{HF}$ at high pressures is unknown. The orthorhombic lattice observed experimentally at ambient conditions[21] was found to be stable in simulations at zero pressure and room temperature. In simulations at pressures of $5 \mathrm{GPa}$ or more, however, we found that the orthorhombic lattice was unstable at room temperature. Since it is difficult to accurately predict the relative energy of molecular solid phases based on density functional theory[22], we took the bcc lattice as a simple representative solid phase without further investigation of other molecular solid lattices. The bcc phase was stable at room temperature for all pressures investigated here.

Initial conditions were generated by creating a bcc unit lattice of two fluorine atoms at the density of interest. The hydrogen positions were optimized within the bcc unit cell. This bcc unit cell was then replicated so the simulation cell contained 54 molecules, and the hydrogen positions were optimized once more. The temperature was increased from $0 \mathrm{~K}$ to $900 \mathrm{~K}$ in steps of 300-450 K, using velocity scaling for ca. 2 ps at each temperature step. All simulations were equilibrated for a minimum of 2 ps at the final temperature of $900 \mathrm{~K}$. Data collection simulations were run for 5-10 ps.

\section{Results and Discussion}

In a molecular phase, the diffusion constants of $\mathrm{H}$ and $\mathrm{F}$ atoms are identical. For a superionic phase, on the other hand, the H atoms are expected to have liquid-like values while the $\mathrm{F}$ atoms do not diffuse. The calculated atomic diffusion constants for our simulations are shown are shown in Fig. 1, with the equation of state results included as the inset plot of Fig. 2. The diffusion constants were determined via the usual method from the slope of the particle 
mean-square displacement at long times, viz., $\left\langle\left(r_{i}(t)-r_{i}(0)\right)^{2}\right\rangle=6 D t$, where $D$ is the diffusion constant.

At 5 and $15 \mathrm{GPa}$ (1.8 and $2.4 \mathrm{~g} / \mathrm{cc}$ ), the bcc lattice melted completely and the system exhibited molecular diffusion (viz., $D_{H} \approx D_{F}$ ). However, at $33 \mathrm{GPa}$ and $66 \mathrm{GPa}(3.0$ and $3.6 \mathrm{~g} / \mathrm{cc}$ ), we observe the onset of a superionic phase, wherein the hydrogens diffuse rapidly over a stable fluorine bcc lattice. For both pressures, the hydrogens exhibit diffusion comparable with molecular diffusion at ambient conditions[23]. Once symmetric hydrogen bonding is achieved at $66 \mathrm{GPa}$ (discussed below), the hydrogen diffusion constant increases substantially to a value above that of the molecular liquid.

Given the relatively short simulations times $(\approx 10 \mathrm{ps})$ accessible by first principles molecular dynamics, it is reasonable to question whether the superionic phase is thermodynamically stable. In order to test the stability of the superionic phase, we heated the bcc lattice to melting at $3.0 \mathrm{~g} / \mathrm{cc}$. We found that in order to melt the bcc lattice the system had to be heated to $1900 \mathrm{~K}$ (in steps of 500 degrees for 2 ps) before melting was observed. The superionic phase thus appears to have a broad region of stability, extending roughly from $900 \mathrm{~K}$ to $1900 \mathrm{~K}$ at $3.0 \mathrm{~g} / \mathrm{cc}$. This observation increases our confidence that the superionic state is thermodynamically stable.

We also found that a superionic phase of $\mathrm{HF}$ can be formed either by heating the molecular solid or by cooling the liquid. We cooled the liquid phase at $3.0 \mathrm{~g} / \mathrm{cc}$ from $1900 \mathrm{~K}$ in steps of 100-200 degrees for $2-4 \mathrm{ps}$. At $600 \mathrm{~K}$ an amorphous superionic solid formed.

The vibrational density of states (VDOS) for the simulations are presented in Figure 2. The VDOS is calculated by taking the Fourier Transform of the velocity autocorrelation function, viz.,

$$
C_{V V}(\omega)=\int\left\langle v_{i}(t) v_{i}(0)\right\rangle e^{-i \omega t} d t
$$

In the molecular liquid at $5 \mathrm{GPa}$, the first peak in the VDOS occurs at ca. $125 \mathrm{~cm}^{-1}$ with a noticeable shoulder at ca. $500 \mathrm{~cm}^{-1}$, both of which correspond closely to translational and librational bands in the experimental Raman spectrum at the same pressure[10] and 25K. In addition, we observe the H-F stretch vibrational band at ca. $3400 \mathrm{~cm}^{-1}$, which matches well with the both the experimental IR [24] spectra and Raman spectra. In contrast, at 66 GPa dramatic changes have occurred within the system. The translational band is absent, and in place of the librational "shoulder" there is a broad band at $600 \mathrm{~cm}^{-1}$. In addition, the $\mathrm{H}-\mathrm{F}$ stretch peak is red-shifted and 
significantly decreased in intensity, a feature also found in the high pressure Raman studies[10]. Similar spectroscopic changes have been attributed to symmetric hydrogen bonding in low temperature, high pressure experiments on various hydrogen bonded systems $[10,11,12,2]$.

The $\mathrm{F}-\mathrm{H}$ radial distribution functions (RDF) at $900 \mathrm{~K}$ and varying pressure are shown in Figure 3. The RDFs were calculated according to the following equation: $g(\mathrm{r}) \equiv \frac{V}{N^{2}}\left\langle\sum_{i, j \neq i}^{N} \delta\left(\mathrm{r}_{\mathrm{ij}}-\mathrm{r}\right)\right\rangle$, where $V$ is the volume of the system, $N$ is the number of particles in the system, and $i$ and $j$ are summed over all species pairs (F-F, H-F, and $\mathrm{H}-\mathrm{H})$. The $g\left(R_{F H}\right)$ exhibits a number of broad peaks similar to a liquid, even though the fluorine atoms occupy lattice positions. This is due to the hopping of hydrogens between specific sites relative to the fluorines, and the relatively high vibrational amplitudes at $900 \mathrm{~K}$. At $5 \mathrm{GPa}$, the first minimum of $g\left(R_{F H}\right)$ is at ca. $1.2 \AA$, indicating the predominance of molecular HF. At $66 \mathrm{GPa}$, the first minimum has shifted to $1.64 \AA$, consistent with hydrogen bond symmetrization. Note that the superionic phase found at $33 \mathrm{GPa}$ shows a first minimum of $g\left(R_{F H}\right)$ of $1.2 \AA$, similar to the molecular liquid. Thus hydrogen bond symmetrization occurs well inside the boundary of the superionic phase.

The first peak at $66 \mathrm{GPa}$ can be decomposed into two Gaussians, one centered at ca. $1 \AA$, and the other at ca. $1.2 \AA$. The first Gaussian most likely corresponds to a covalent bond, and the peak at $1.2 \AA$ to an ionic bond with the neighboring fluorine. Thus, the hydrogen bonding in superionic HF at $66 \mathrm{GPa}$ is not entirely symmetrized in that the covalent and ionic bond lengths are not yet equal.

Coordination numbers clearly demonstrate the symmetrization of hydrogen bonding in superionic HF. The coordination number is defined as the volumetric integral of $g\left(R_{F H}\right)$ from 0 to the first minimum. In Figure 4 the coordination number is seen to clearly change from 1 at $5 \mathrm{GPa}$, consistent with molecular HF, to 2 at $66 \mathrm{GPa}$, consistent with symmetrically hydrogen bonded HF. The coordination number undergoes a continuous change with pressure, despite the first order phase transition from the liquid phase to the superionic phase.

The behavior of the coordination number in $\mathrm{HF}$ is very similar to that found in previous simulations of $\mathrm{H}_{2} \mathrm{O}[3]$. In Figure 5 we show the coordination number of $\mathrm{O}$ in $\mathrm{H}_{2} \mathrm{O}$ at $2000 \mathrm{~K}$ as a function of pressure. The coordination increases from 2 in molecular $\mathrm{H}_{2} \mathrm{O}$ to 4 in superionic $\mathrm{H}_{2} \mathrm{O}$. As with $\mathrm{HF}$, the transformation to symmetric hydrogen bonding occurs well inside 
the superionic phase boundary.

The change from asymmetric to symmetric hydrogen bonding in the superionic phase could occur either as a continuous transformation within the superionic phase or as a distinct thermodynamic phase transition. Although the limited number of simulations presented here do not fully address this issue, the continuous nature of the equation of state curve in Figure 2 suggest a continuous tranformation.

It is difficult to unambiguously determine chemical species in highly reactive condensed phase materials. We take two simple approaches here. In the first, we define instantaneous species based on the $\mathrm{F}-\mathrm{H}$ bond distance. If the bond distance is less than a value $r_{c}$, we count the atom pair as bonded. Determining all the bonds in the system gives the chemical species at each point in time. Considering the $\mathrm{F}-\mathrm{H}$ vibrational frequency at $3400 \mathrm{~cm}^{-1}$, species with lifetimes less than an $\mathrm{F}-\mathrm{H}$ bond vibrational period (10 fs) are "transient", and do not represent bound molecules. The optimal cutoff $r_{c}$ between bonded and non-bonded species is given by the location of the maximum in the free energy surface, viz. $W(r)=-k_{B} T \ln \left[g\left(R_{F H}\right)\right]$, where $W(r)$ is the free energy surface (potential of mean force). The maximum in $W(r)$ corresponds to the first minimum in $g\left(R_{F H}\right)$. This choice, while intuitive, also corresponds to the optimal choice of a transition state for the dissociation[25] within transition state theory. In the second method to determine species, we count two atoms as bonded only if their distance is less than $r_{c}$ for a given period of time. This has the advantage of reducing possible overbonding in the chemical picture, but has the disadvantage of introducing an empirical parameter in the description, i.e. the minimum bond lifetime.

We now analyze the molecular species, lifetimes and structures using the first method. We find that no molecular hydrogen $\left(\mathrm{H}_{2}\right)$ or molecular fluorine $\left(\mathrm{F}_{2}\right)$ has been formed during the simulations at all densities. Snapshots from several of the simulations are shown in Fig. 6. At $5 \mathrm{GPa}$, the predominant species is HF, which has a lifetime of close to 1 ps. We also observed clustering wherein up to three HF molecules have formed neutral and ionic clusters. However, all of these clusters have lifetimes less than $10 \mathrm{fs}$, indicating that they are not bound complexes. At $15 \mathrm{GPa}$, the HF lifetime has dropped to less than 150 fs. For this density, clusters up to the neutral HF hexamer were found. At $33 \mathrm{GPa}$ we have observed the superionic phase. At this point, the HF lifetime has decreased to ca. 72 fs. The degree of clustering has become more pronounced, with greater concentrations of up to the neutral and ionic hexamer being recorded. The predominant species at all of these state points 
is HF, and all other species detected had lifetimes of less than $10 \mathrm{fs}$.

Our result for $66 \mathrm{GPa}$ is the most interesting state point investigated. At this density, the predominant species is a transient network of $\mathrm{F}-\mathrm{H}$ bonds, encompassing most if not all of the atoms in the simulation. This network is due to the increase in $\mathrm{F}-\mathrm{H}$ bond distance; no $\mathrm{F}-\mathrm{F}$ or $\mathrm{H}-\mathrm{H}$ bonds were found at any point during the simulation. Many of the $\mathrm{F}-\mathrm{H}$ pairs that are considered bonded are fleeting in nature. Thus, we have repeated our calculations with the second method of species determination, considering only bonds with lifetimes greater than a 10 fs cutoff. 10 fs corresponds roughly to one vibrational period of the F-H bond, and thus is a logical minimal bond lifetime.

We observed ca. $60 \%$ of $\mathrm{H}-\mathrm{F}$ bonds that met our distance criteria also met this lifetime criteria at $66 \mathrm{GPa}$. The average lifetime of these bonds was ca. $160 \mathrm{fs}$. Inspection of $10 \mathrm{fs}$ long segments of the trajectory revealed long hydrogen bond chains, somewhat similar to what is observed in the liquid at much lower temperatures and pressure[13]. Several stable HF molecules were also observed with the above criteria, although the predominant species was the hydrogen bonded network. Observation of the hydrogen bond chains did not depend particularly on the lifetime cutoff used. For example, ca. $50 \%$ of bonds met an increased lifetime cutoff of $20 \mathrm{fs}$, and ca. $30 \%$ met a cutoff of 50 fs. Consequently, this extended hydrogen bond network is a (relatively) long-lived feature of hydrogen bonded superionic solids, that is present for reasons other than fleeting contact between nuclei.

We find that at $66 \mathrm{GPa}$, hydrogens diffuse via a trigonal coordination mechanism. This is consistent with features of well-known superionic solids, such as AgI, in which the silver ion diffuses through the trigonal interstitial sites[4]. Snapshots of a hydrogen diffusing through the lattice are shown in Fig. 7. The formation of three bonds likely weakens each individual one, thus allowing the hydrogen to hop more easily through the fluorine sublattice. The ease with which multiple ionic bonds can form is due to the symmetric hydrogen bonding.

In order to determine the degree of covalency of $\mathrm{H}-\mathrm{F}$ bonds in our simulations, the maximally localized Wannier centers[26] of several trajectories were calculated, and a distribution function of the distance from the nearest fluorine atom was determined. There is a minimum in the distribution function $\left(r_{\min }\right)$ at roughly $0.35 \AA$ in all of the simulations. We used $r_{\min }$ to differentiate between Wannier centers corresponding to lone pairs $\left(r<r_{\min }\right)$ and those corresponding to covalent bonds $\left(r>r_{\min }\right)$. This definition was 
used to compute the percentage of ionic and covalent bonds. At pressures of $5-33 \mathrm{GPa}$ we find that $96-100 \%$ of the $\mathrm{F}-\mathrm{H}$ bonds are covalent. For 66 GPa, we find that about $50 \%$ of the bonds are covalent.

Finally, we have performed a Mulliken[27] population analysis of a snapshot of the simulations at $66 \mathrm{GPa}$ in order to estimate the ionic charges. There are difficulties in applying charge localization schemes with a planewave basis set, since a secondary atom-centered basis set must be introduced during the charge localization. In order to generate a simple estimate of Mulliken charges, the mixed basis set CP2K[28] code was used to perform the Mulliken population analysis on a representative snapshot from the CPMD simulation at $66 \mathrm{GPa}$, with a cutoff of $400 \mathrm{Ry}$, a basis set of DZVP, and with the BLYP functional[28, 29].

For a single snapshot, we observe an average hydrogen charge (in au) of $+0.43 \pm 0.033$ and an average fluorine charge of $-0.43 \pm 0.049$. Interestingly, we observed that a geometry optimized HF monomer has nearly identical charges on the atomic species. Considering the observed $50 \%$ covalent bonding, we expect that the site charges will approach an absolute value of 0.5 as the hydrogen bonding becomes increasingly symmetric. $\mathrm{n}$

\section{Conclusions}

We have predicted a new superionic solid phase of HF. The superionic phase is characterized by a stable fluorine bcc lattice and highly mobile hydrogen atoms. At the highest pressures simulated, we see a symmetrization of the hydrogen bond, where the superionic solid becomes an extended network of very short-lived partially covalent bonds. The hydrogens diffuse through the fluorine sub-lattice via trigonal interstitial sites. It should be observable by experiments at ca. $33 \mathrm{GPa}$ and $900 \mathrm{~K}$ (c. f. $49 \mathrm{GPa}$ and $1000 \mathrm{~K}$ for water[2]). In addition, the hydrogens and fluorines are not fully ionized, similar to previous results for a superionic solid at ambient pressure[30].

The symmetrically hydrogen bonded form of the superionic solid is predicted to occur at $66 \mathrm{GPa}$ (c. f. $95 \mathrm{GPa}, 2000 \mathrm{~K}$ for water[3]). There is some reason to expect that the experimental observation of symmetric hydrogen bonding in superionic HF could be technically easier than the corresponding observation in $\mathrm{H}_{2} \mathrm{O}$. Thus, $\mathrm{HF}$ serves as an excellent model for studying the role of symmetric hydrogen bonding in high pressure superionic solids.

Future simulations may explore the role of quantum effects in superionic 
solid phases. We expect that zero point motion will favor hydrogen delocalization, perhaps leading to a larger calculated locus for the superionic phase. Such simulations would be extremely difficult computationally, but could be accessed with the most recent terascale computers.

\section{Acknowledgments}

This work was performed under the auspices of the U. S. Department of Energy by the University of California Lawrence Livermore National Laboratory under contract No. W-7405-Eng-48. We thank Will Kuo and Chris Mundy for helpful discussions. Computations were performed at Lawrence Livermore National Laboratory using the following massively parallel computers: Multiprogrammatic Capability Resource, Thunder, UV, and Frost. We also used the Seaborg IBM cluster at the National Energy Research Scientific Computing Center.

\section{References}

[1] Cavazzoni, C., Chiarotti, G. L., Scandolo, S., Tosatti, E., Bernasconi, M., \& Parrinello, M. (1999) Science 283, 44-46.

[2] Goncharov, A. F., Goldman, N., Fried, L. E., Crowhurst, J. C., Kuo, I.-F. W., Mundy, C. J., \& Zaug, J. M. (2005) Phys. Rev. Lett. 94(12), 125508 .

[3] Goldman, N., Fried, L. E., Kuo, I.-F. W., \& Mundy, C. J. (2005) Phys. Rev. Lett. 94(21), 217801.

[4] Hull, Stephen (2004) Rep. Prog. Phys. 67, 1233-1314.

[5] Demontis, P., LeSar, R., \& Klein, M. L. (1988) Phys. Rev. Lett. 60(22), 2284 .

[6] Schwager, B., Chudinovbskikh, L., \& Boehler, R. (2004) J. Phys.: Condens. Matter 16, 1177.

[7] Lin, J.-F., Gregoryanz, E., Struzhkin, V. V., Somayazulu, M., k.Mao, H., \& Hemley, R. J. (2005) Geophys. Res. Lett. 32, 11306. 
[8] Hemley, R. J. (2000) Annu. Rev. Phys. Chem. 51, 763.

[9] Dyke, J. R., Howard, B. J., \& Klemperer, W. (1972) J. Chem. Phys. 56, 2442.

[10] Pinnick, D. A., Katz, A. I., \& Hanson, R. C. (1989) Phys. Rev. B 39, 8677.

[11] Katoh, E., Yamawaki, H., Fujihisa, H., Sakashita, M., \& Aoki, K. (2000) Phys. Rev. B 61, 119.

[12] Ikeda, T., Sprik, M., Terakura, K., \& Parrinello, M. (1999) J. Chem. Phys. 111, 1595-1607.

[13] Röthlisberger, U. \& Parrinello, M. (1997) J. Chem. Phys. 106, 4658.

[14] Kreitmeir, M., Bertagnolli, H., Mortensen, J. J., \& Parrinello, M. (2003) J. Chem. Phys. 118, 3639.

[15] Car, R. \& Parrinello, M. (1985) Phys. Rev. Lett. 55(22), 2471-2474, CPMD, version 3.91, copyright IBM Corp., 1990-2004, copyright MPI für Festkörperforschung Stuttgart, 1997-2001, http://www.cpmd.org.

[16] Becke, A. D. (1988) Phys. Rev. A 38, 3098.

[17] Lee, C., Yang, W., \& Parr, R. G. (1988) Phys. Rev. B 37, 785.

[18] Troullier, N. \& Martins, J. (1991) Phys. Rev. B 43, 1993.

[19] Nosé, S. (1984) Mol. Phys. 52, 255.

[20] Hoover, W. G. (1985) Phys. Rev. A 31, 1695.

[21] Johnson, M. W., Sándor, E., \& Arzi, E. (1975) Acta Cryst. B31, 1998.

[22] Byrd, E. F. C., Scuseria, G. E., \& Chabalowski, C. F. (2004) J. Phys. Chem. B 108, 13100-13106.

[23] Karger, N., Vardag, T., \& Lüdemann, H.-D. (1994) J. Chem. Phys. 100, 8271.

[24] Buslov, D. K., Sushko, N. I., \& Yukhnevich, G. V. (2003) J. Opt. Tech. $70,28$. 
[25] Chandler, D. (1978) J. Chem. Phys. 68(6), 2959-2970.

[26] Silvestrelli, P. L. \& Parrinello, M. (1999) Phys. Rev. Lett. 82, 3308.

[27] Mulliken, R. S. (1955) J. Chem. Phys. 23, 1833.

[28] For information of the CP2K code see http://www.berlios.de.

[29] VandeVondele, J., Krack, M., Mohamed, F., Parrinello, M., Chassaing, T., \& Hutter, J. (2005) Comp. Phys. Comm. 167, 103.

[30] Shimojo, F. \& Aniya, M. (2003) J. Phys. Soc. Japan 72, 2702. 


\section{Figures}

- Figure 1: Hydrogen and fluorine diffusion constants as a function of density. The onset of symmetric hydrogen bonding is discussed further in the text.

- Figure 2: Vibrational density of states at simulations at 5, 33 and 66 GPa. The equation of state is in the inset.

- Figure 3: F-H Radial distribution functions.

- Figure 4: Coordination number of $\mathrm{F}$ as a function of pressure in HF at $900 \mathrm{~K}$.

- Figure 5: Coordination number of $\mathrm{O}$ as a function of pressure in $\mathrm{H}_{2} \mathrm{O}$ at $2000 \mathrm{~K}$

- Figure 6: Snapshots of the simulations at 5, 33, and 66 GPa. The fluorine atoms are colored green and the hydrogens grey. Bonds were only drawn if they met both the length and lifetime cutoffs (1.63 $\AA$ and 10 fs, respectively). Dashed lines correspond to hydrogen bonds that have lifetimes longer than the 10 fs cutoff. The snapshots at 5 and $33 \mathrm{GPa}$ exhibits chains of hydrogen bonds, similar to what is found in the ambient liquid[13, 14]. The bcc lattice in the 33 and $66 \mathrm{GPa}$ simulations is clearly observed. Several $\mathrm{H}_{2} \mathrm{~F}$ species can be seen in the 66 GPa picture, which are artifacts of the periodic boundary conditions. The superionic solid at 66 GPa exhibits superionic diffusion with near symmetric hydrogen bonding.

- Figure 7: Sequential snapshots of superionic HF, taken from the simulation at $66 \mathrm{GPa}$. Fluorines are labeled green and the hydrogens are white. In (b), a triply coordinated hydrogen is found. The blue bonds indicate the activated nature of the configuration. This transition state configuration has a lifetime of only ca. 10 fs. Part (c) shows the new $\mathrm{H}-\mathrm{F}-\mathrm{H}$ bond pair. 


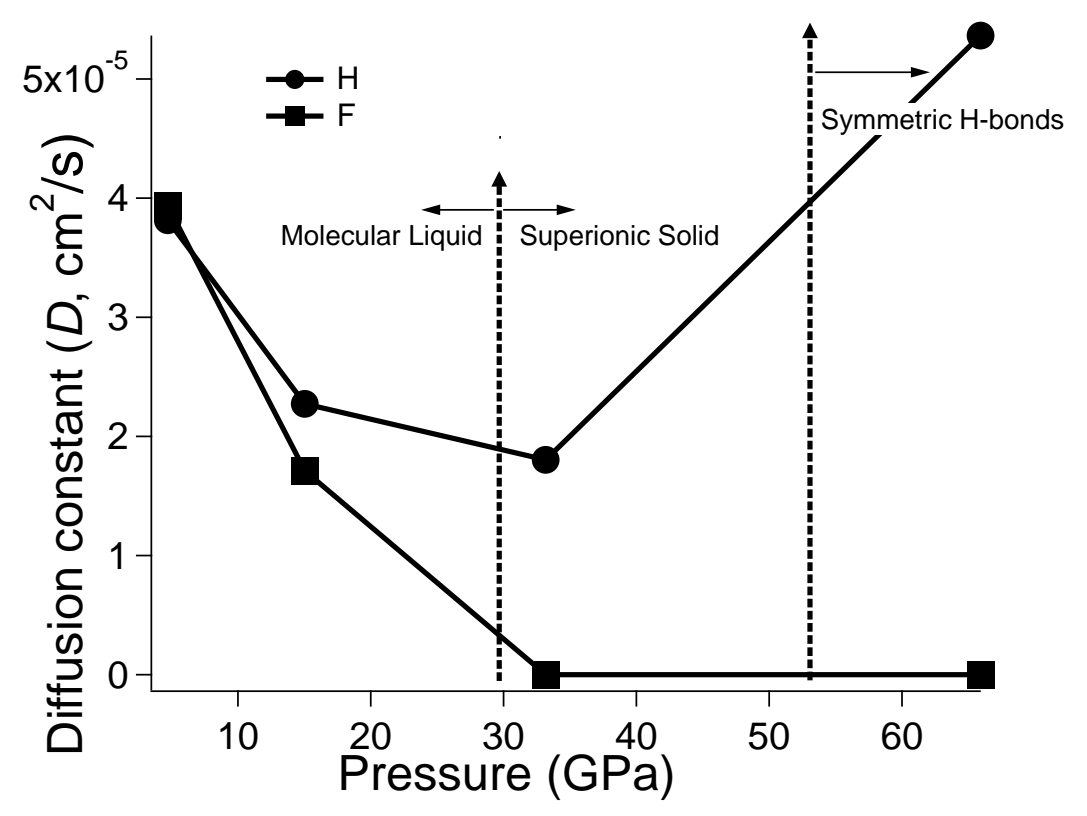

Figure 1: 


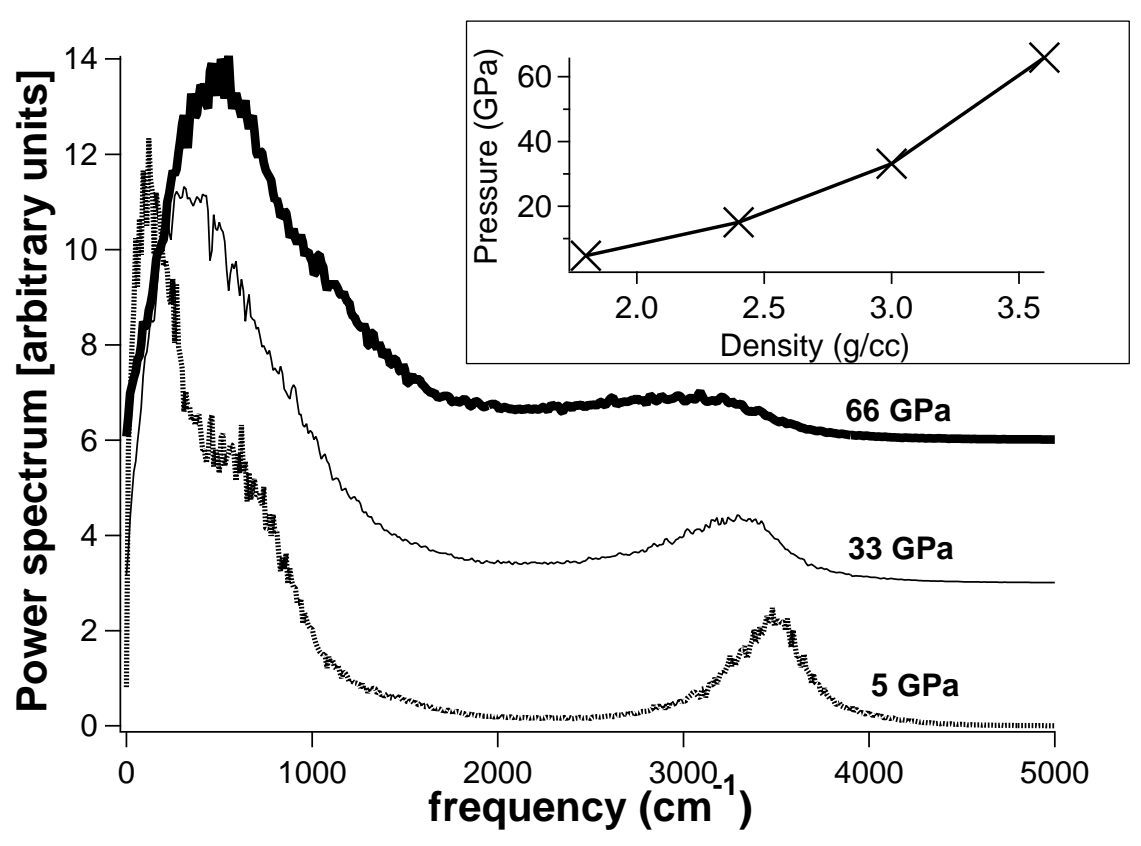

Figure 2:

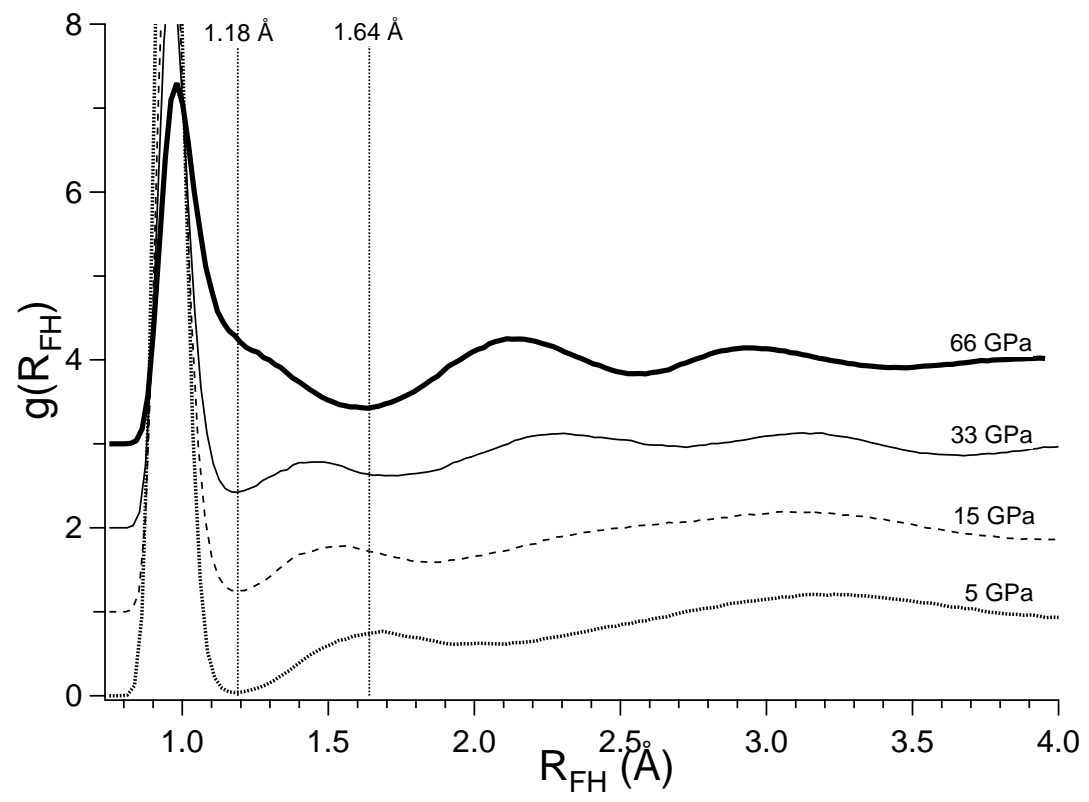

Figure 3: 


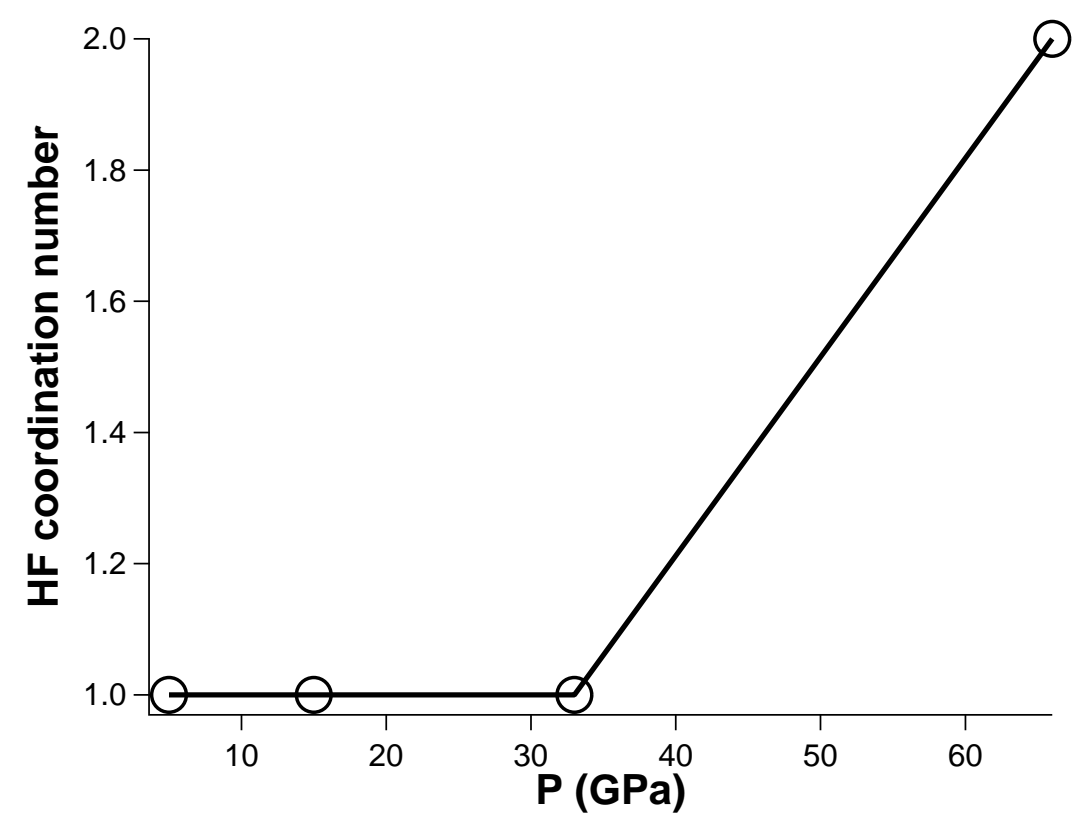

Figure 4:

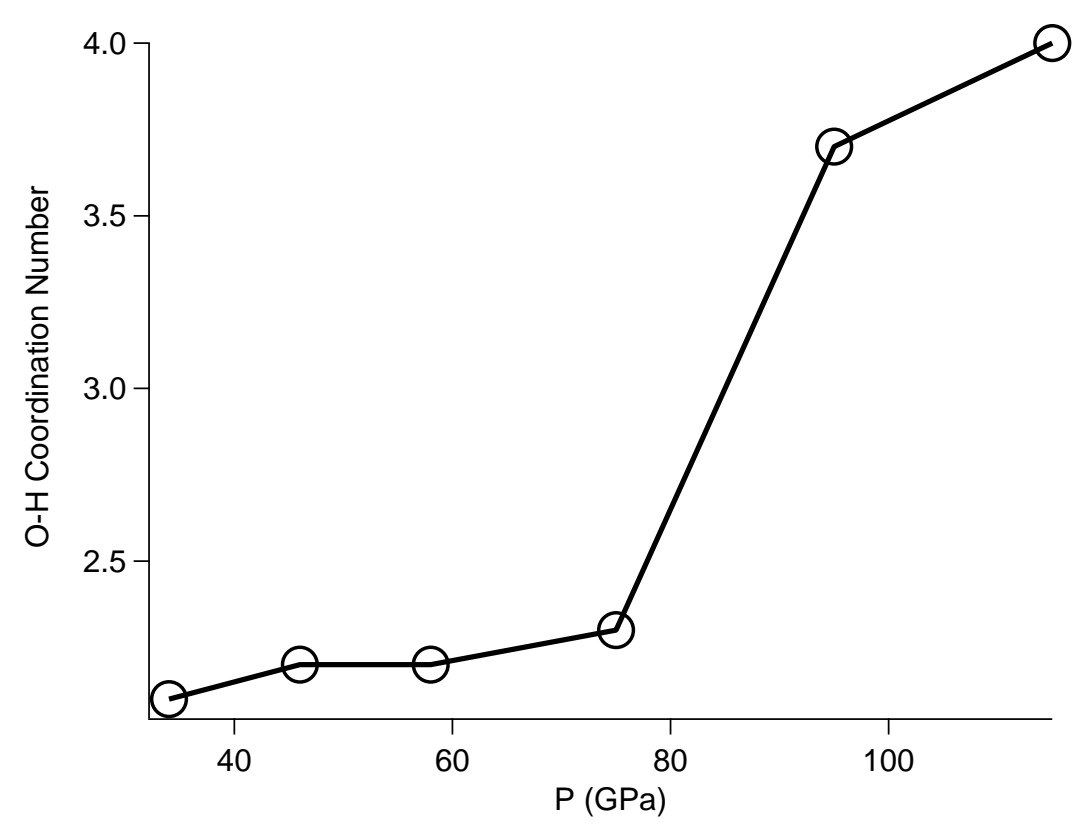

Figure 5: 


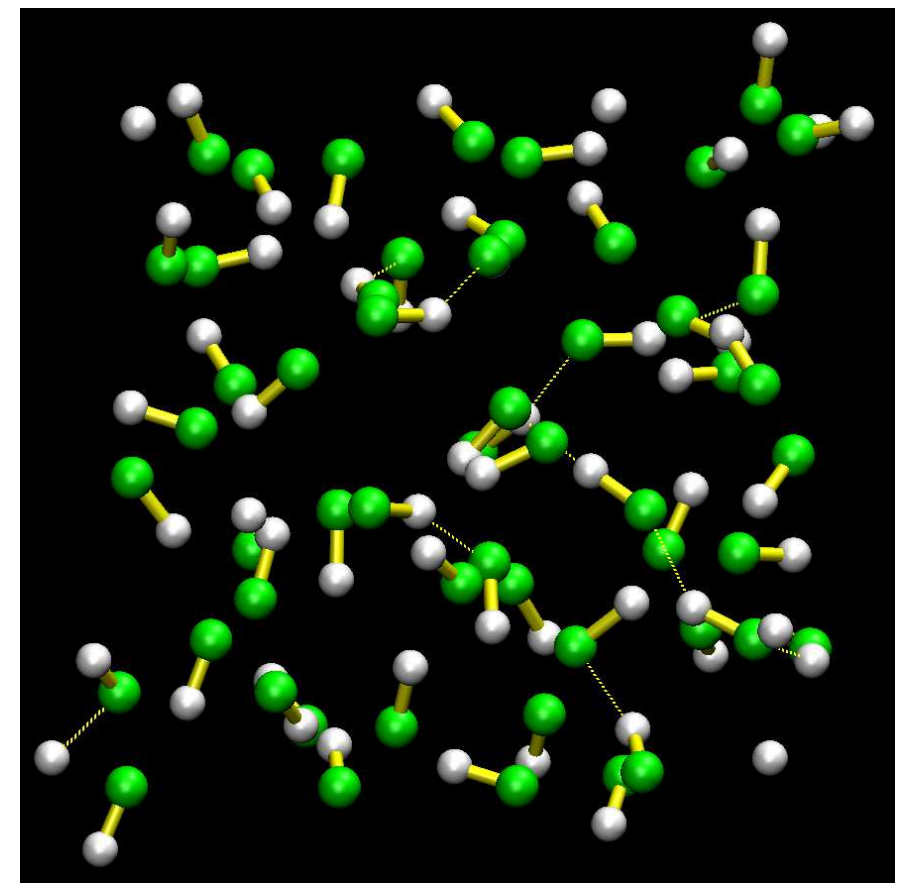

(a) Molecular liquid.

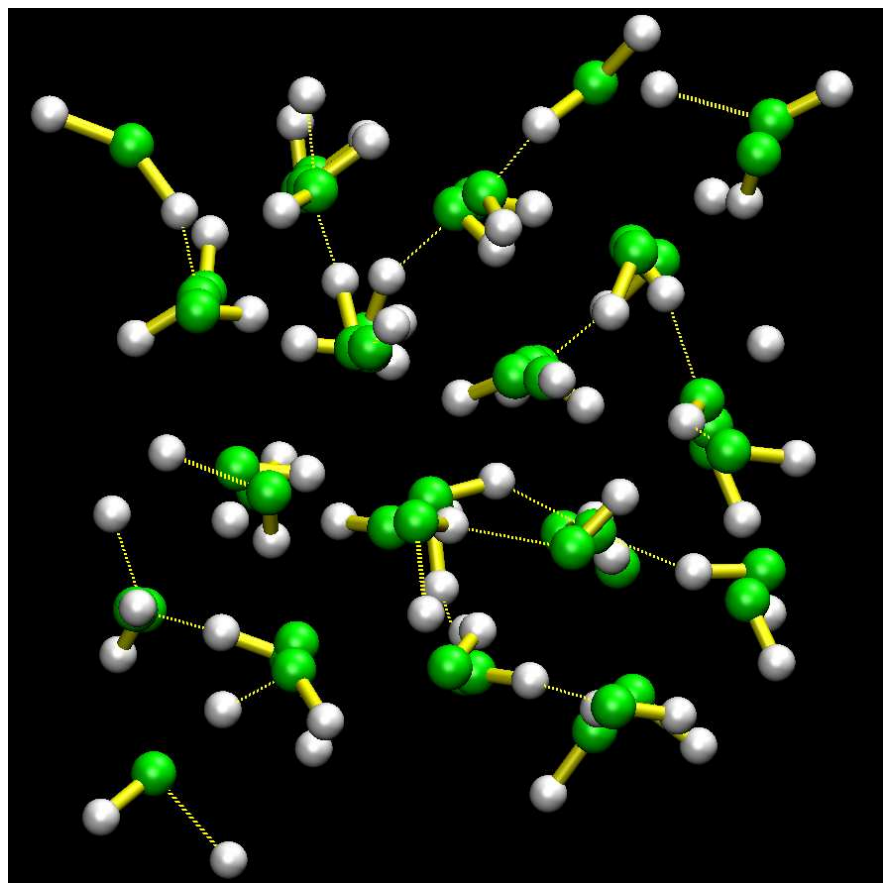

(b) Asymmetric H-bonded superionic solid.

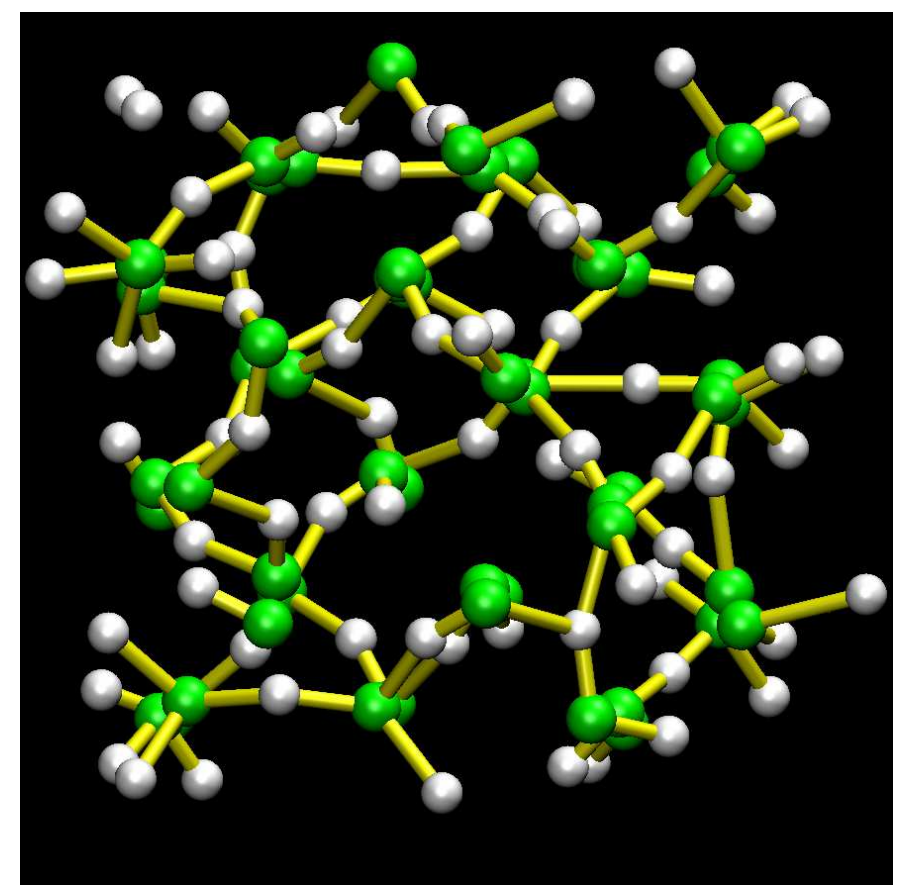

(c) Symmetric H-bonded superionic solid.

Figure 6: 


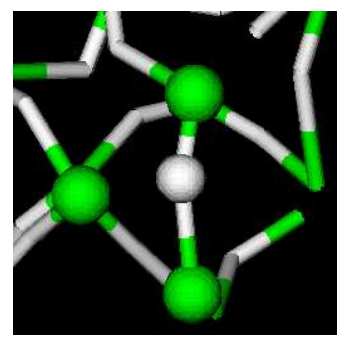

(a)

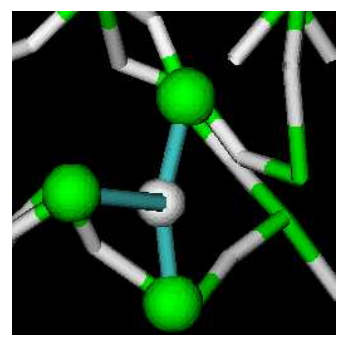

(b)

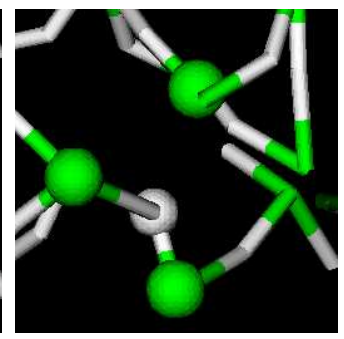

(c)

Figure 7: 